Submitted to Physical Review Letters

\title{
Many-Body Corrections to Charged-Current Neutrino Absorption Rates in Nuclear Matter
}

\author{
Adam Burrows \\ Department of Astronomy, The University of Arizona, Tucson, AZ 85721 \\ e-mail: burrows@as.arizona.edu \\ R. F. Sawyer \\ Department of Physics, The University of California, Santa Barbara, CA 93106 \\ e-mail: sawyer@sarek.physics.ucsb.edu
}

\begin{abstract}
Including nucleon-nucleon correlations due to both Fermi statistics and nuclear forces, we have developed a general formalism for calculating the charged-current neutrino-nucleon absorption rates in nuclear matter. We find that at one half nuclear density many-body effects alone suppress the rates by a factor of two and that the suppression factors increase to $\sim 5$ at $4 \times 10^{14} \mathrm{~g} \mathrm{~cm}^{-3}$. The associated increase in the neutrino-matter meanfree-paths parallels that found for neutral-current interactions and opens up interesting possibilities in the context of the delayed supernova mechanism and protoneutron star cooling.
\end{abstract}




\section{INTRODUCTION}

The neutrino absorption and scattering opacities in the post-shock core of a supernova, in which nuclei are largely disintegrated into nucleons, determine the duration, spectrum, and flavor distribution of the emerging neutrino pulse. It has been known for some time that the interactions among nucleons in the denser regions can change these opacities significantly, but to date there has been no comprehensive treatment given in the literature and present calculations of the complete supernova process do not include the effects of interactions on the opacities.

The neutrino-matter interaction rates can be related to the space- and time-dependent correlations among the set of density operators for the separate nuclear constituents (to find the Gamow-Teller parts we must consider separate spin-up and spin-down densities). In the case of neutral-current interactions [1], there is an instructive limit, which also provides an estimate of the effects, in which the combined limits of large nucleon mass and small neutrino energy allow the use of long-wavelength limits of equal-time correlation functions, in turn expressible in terms of the second derivatives of an energy density functional with respect to various densities. This approach is the direct multichannel generalization of the familiar results for light scattering from the thermal density fluctuations in a fluid, where it is the compressibility that determines the long-wavelength opacity, and it was used in references [2] and [3] to find significant reductions of neutral-current opacity in certain regions. In Burrows \& Sawyer [1] , an approach based on ring graphs was used to encompass these results and to extend them to domains in which the equal-time and long-wavelength limits are not clearly applicable.

The use of the equal-time and long-wavelength limits to express correlation functions in terms of static susceptibilities cannot be extended to the charged-current interactions when there is a large chemical potential difference between protons and neutrons. Furthermore, there do not exist in the present literature systematic estimates of the effects of interactions on the charged-current opacities for electron neutrinos. In the present work, we give a theoretical framework for addressing these opacities, based on summing ring graphs, together with the results of calculations with input parameters taken from the current phenomenology of nuclear matter.

\section{MANY-BODY FORMALISM FOR CHARGED-CURRENT RATES}

The charged-current interactions of the nuclear medium are determined by the statistical

averages involving the nucleonic charged-current operator, $j_{\mu}=\bar{\psi}_{p} \gamma_{\mu}\left(1-g_{A} \gamma_{5}\right) \psi_{n}$, and its Hermitean conjugate,

$$
W_{\mu \nu}(q, \omega)=-i Z^{-1} \int d^{4} x e^{-i \mathbf{q} \cdot \mathbf{x}} e^{i(\omega+\hat{\mu}) t} \operatorname{Tr}\left[e^{-\beta\left(H-\Sigma \mu_{i} N_{i}\right)}\left[j_{\mu}(\mathbf{x}, t), j_{\nu}^{\dagger}(0,0)\right]\right] \theta(t),
$$

where $Z$ is the partition function and $\hat{\mu}$ is the chemical potential difference, $\hat{\mu}=\mu_{n}-\mu_{p}$. The vector, $(\mathbf{q}, \omega)$, is the momentum-energy transfer from the leptons to the medium. The transition rate, differential in angle and $\omega$, for the reaction $\nu_{e}+n \rightarrow e^{-}+p$ is given in terms of $W_{\mu \nu}$ as 


$$
\frac{d^{2} \Gamma\left(\nu_{e} \rightarrow e^{-}\right)}{d \omega d \cos (\theta)}=\left(2 \pi^{2}\right)^{-1} G_{W}^{2} \frac{\left(E_{1}-\omega\right)^{2}\left[1-f_{e}\left(E_{1}-\omega\right)\right]}{1-\exp [-\beta(\omega+\hat{\mu})]} \Lambda^{\mu \nu}(q, \omega) \operatorname{Im} W_{\mu \nu}(q, \omega)
$$

where

$$
\Lambda^{\mu \nu}=\left(4 E_{1} E_{2}\right)^{-1} \operatorname{Tr}\left[\not p_{1}\left(1-\gamma_{5}\right) \gamma^{\mu} \not p_{2} \gamma^{\nu}\left(1-\gamma_{5}\right)\right]
$$

$q=\left[E_{1}^{2}+\left(E_{1}-\omega\right)^{2}-2 E_{1}\left(E_{1}-\omega\right) \cos \theta\right]^{1 / 2}$, and $E_{1}$ in the incident neutrino energy. To verify the factors in (2) replace the commutator in (1) by the unordered product, the $\theta(t)$ function by $1 / 2$ in order to capture the imaginary part, and the statistical factor in the denominator by unity. Then, the introduction of a complete set of states between the current operators gives the inclusive $\nu_{e} \rightarrow e^{-}$rate. Recalling that the Heisenberg picture for the density operators is defined with respect to the operator $H-\Sigma \mu_{i} N_{i}$, we note that the $e^{i \hat{\mu} t}$ factor in (1) will be canceled before the time integral is performed. The other term in the commutator gives the same answer, but with an additional factor, $-\exp [-\beta(\omega+\hat{\mu})]$. The statistical factor in the denominator of (2) cancels when the two terms are combined.

Taking the nucleons as nonrelativistic, which is allowable up to about twice nuclear density, we note that the components $W_{i 0}$ and $W_{0 i}$ vanish, and that the space tensor, $W_{i j}$, derives from the axial-vector current and can be written in the form,

$$
W_{i j}(\omega, \cos \theta)=g_{A}^{2} W_{A}(\omega, \cos \theta) \delta_{i j}+g_{A}^{2} W_{T}(\omega, \cos \theta) q_{i} q_{j}
$$

We define $W_{00}=W_{V}$, as it comes entirely from the vector current. Combining with (3) and calculating the lepton trace, we now have

$$
W_{\mu \nu} \Lambda^{\mu \nu}=2(1+\cos \theta) W_{V}+2(3-\cos \theta) g_{A}^{2} W_{A}+2 \omega^{2}(1+\cos \theta) g_{A}^{2} W_{T} .
$$

We calculate the three functions $W_{V}, W_{A}$, and $W_{T}$ in the ring approximation. For these graphs, a unit of charge is passed in each nuclear interaction in the expansion of the chargedcurrent correlation function of (目). If the potential between nucleons $a$ and $b$ conserves isotopic spin, this means that only the terms containing the isotopic operator $\vec{\tau}^{(a)} \cdot \vec{\tau}^{(b)}$ enter. We assume a potential in momentum space,

$$
V^{a, b}(q, \omega)=\vec{\tau}^{a} \cdot \vec{\tau}^{b}\left[v_{1}(q)+\vec{\sigma}^{a} \cdot \vec{\sigma}^{b} v_{2}(q)+\vec{\sigma}^{a} \cdot \vec{q} \vec{\sigma}^{b} \cdot \vec{q} v_{3}(q, \omega)\right]
$$

We have inserted in the $v_{3}$ term a dependence on energy transfer, $\omega$, in order to accomodate the one-pion exchange force,

$$
v_{3}(q, \omega)=-f^{2} m_{\pi}^{-2}\left(q^{2}+m_{\pi}^{2}-\omega^{2}\right)^{-1}
$$

with $f^{2} \approx 1$.

For the other two potentials, we follow the development of [1] in taking zero-range forms fitted to the Landau parameters of reference [四] and obtain $v_{2}=3.4 \mathrm{~m} / \mathrm{m}^{*} \times 10^{-5}(\mathrm{MeV})^{-2}$ and $v_{1}=1.88 \mathrm{~m} / \mathrm{m}^{*} \times 10^{-5}(\mathrm{MeV})^{-2}$, where $\mathrm{m}^{*}$ is the effective nucleon mass in the medium. We are assuming isospin invariant forms, despite the fact that our application will be to unsymmetric matter. This appears to us to be the state of the art. It is not totally satisfactory, in view of the fact that the $v_{1}$ and $v_{2}$ terms are phenomenological forms that obtain in nuclear matter rather than forms based on elementary meson exchanges. Note that in 
the case of non-symmetric matter isospin symmetry is still broken through the polarization functions. We define the nucleon-charge-raising polarization part, $\Pi(q, \omega)$, in parallel with (1), in terms of the retarded commutator of the density $n_{c}=\psi_{p}^{\dagger} \psi_{n}$ and its Hermitean conjugate,

$$
\Pi(q, \omega)=-i Z^{-1} \int d^{4} x e^{-i \mathbf{q} \cdot \mathbf{x}} e^{i(\omega+\hat{\mu}) t} \operatorname{Tr}\left[e^{-\beta\left(H-\Sigma \mu_{i} N_{i}\right)}\left[n_{c}(\mathbf{x}, t), n_{c}^{\dagger}(0,0)\right]\right] \theta(t) .
$$

Note that the function that gives the vector part of the rate, $W_{V}(q, \omega)$, is exactly given by П. The ring approximation [5] for $\Pi$ is now

$$
W_{V}(q, \omega)=\Pi(q, \omega)=\frac{\Pi^{(0)}(q, \omega)}{1-2 v_{1}(q) \Pi^{(0)}(q, \omega)},
$$

where $\Pi^{(0)}$ is the polarization function in the absence of interactions. The factor of 2 multiplying the potential comes from the isospin operator in the potential (6). The spin dependent parts of the potential do not contribute.

For the axial contribution, the operative non-relativistic forms of the current are the operators $n_{c}^{i}=\psi_{p}^{\dagger} \sigma^{i} \psi_{n}$, and the analogue to (\$) will be a tensor with indices $i, j$. In the absence of interactions, this tensor is given by $\delta_{i, j} \Pi^{(0)}$. Thus, summing the axial chain to get $W_{A}(q, \omega)$ we obtain exactly the same structure as (8) with $v_{2}$ replacing $v_{1}$, but with the same function $\Pi^{(0)}$,

$$
W_{A}(q, \omega)=\frac{\Pi^{(0)}(q, \omega)}{1-2 v_{2}(q) \Pi^{(0)}(q, \omega)} .
$$

The potential $v_{3}$ does not enter (10), even though it couples to the axial-vector terms, since any ring graph chain in which at least one $v_{3}$ participates becomes a contribution to $W_{T}$. Elementary combinatorics for the tensor chain gives,

$$
W_{T}(q, \omega)=\frac{\left[\Pi^{(0)}(q, \omega)\right]^{2} v_{3}(q)}{1-2\left[v_{2}(q)+q^{2} v_{3}(q, \omega)\right] \Pi^{(0)}(k, \omega)} .
$$

The requisite polarization function is given by

$$
\Pi^{(0)}(q, \omega)=-2 \int \frac{d^{3} p}{(2 \pi)^{3}} \frac{f\left(|\mathbf{p}|, \mu_{n}\right)-f\left(|\mathbf{p}+\mathbf{q}|, \mu_{p}\right)}{\omega+\epsilon_{\mathbf{p}}-\epsilon_{\mathbf{p}+\mathbf{q}}+i \eta},
$$

where the functions $f$ are the nuclear Fermi occupation functions for the indicated momenta and chemical potentials. We conceptually extend (12) to include the average potentials that the nucleons experience in the medium, $v_{p, n}$, by making the replacements $(\mathbf{p})^{2} /(2 m) \rightarrow$ $(\mathbf{p})^{2} /(2 m)+v_{n}$ and $(\mathbf{p}+\mathbf{q})^{2} /(2 m) \rightarrow(\mathbf{p}+\mathbf{q})^{2} /(2 m)+v_{p}$, both in the denominator and in the distribution functions in the numerator. However, we suppose that we are starting with a table of densities and temperatures $(T)$ from an equation of state that already takes into account the potentials $v_{p, n}$. If we utilize Fermi distributions in which the chemical potentials are derived from the input densities using the free-particle relations, these average potential corrections are automatically included and the parameters $v_{p, n}$ do not appear explicitly in the formalism. To do the computations, we use the following form for $\Pi^{(0)}$ : 


$$
\begin{array}{r}
\Pi^{(0)}(q, \omega)=\frac{m^{2}}{2 \pi^{2} q \beta}\left(\int_{-\infty-i \epsilon}^{\infty-i \epsilon} d s s^{-1} \log \left[1+e^{-\left(s+Q_{+}\right)^{2}+\beta \mu_{n}}\right]\right. \\
\left.+\int_{-\infty+i \epsilon}^{\infty+i \epsilon} d s s^{-1} \log \left[1+e^{-\left(s+Q_{-}\right)^{2}+\beta \mu_{p}}\right]\right)
\end{array}
$$

where

$$
Q_{ \pm}=\left(\frac{m \beta}{2}\right)^{1 / 2}\left(\mp \frac{\omega}{q}+\frac{q}{2 m}\right)
$$

which gives

$$
\operatorname{Im} \Pi^{(0)}(q, \omega)=\frac{m^{2}}{2 \pi \beta q} \log \left[\frac{1+e^{-Q_{+}^{2}+\beta \mu_{n}}}{1+e^{-Q_{+}^{2}+\beta \mu_{p}-\beta \omega}}\right]
$$

and

$$
\operatorname{Re} \Pi^{(0)}(q, \omega)=\frac{m^{2}}{2 \pi^{2} q \beta} \int_{0}^{\infty} \frac{d s}{s} \log \left[\frac{1+e^{-\left(s+Q_{+}\right)^{2}+\beta \mu_{n}}}{1+e^{-\left(s-Q_{+}\right)^{2}+\beta \mu_{n}}}\right]+\left(\omega \rightarrow-\omega, \mu_{n} \rightarrow \mu_{p}\right) .
$$

The imaginary part of the polarization (15) can be obtained by direct integration, and is the same as that given in references [1.6]. Given this, the full function (13) can be verified by checking the analytic properties in the $\omega$ plane.

We look at the results of medium interactions for two sets of conditions typical of the dense interior of a protoneutron star, but at different times; first for an early time $(t<5$ seconds), when the lepton number is large and second at a later time ( $t>10$ seconds), after which the lepton excess has largely radiated away [7].

\section{LEPTON-RICH ERA}

The densities of the various species are such that at zero temperature the inequality $2 p_{F}^{(p)}>\left|p_{F}^{(n)}-p_{F}^{(\nu)}\right|$ holds. This inequality allows the single nucleon process to proceed at full strength. That is to say, at low temperatures the function $\operatorname{Im}^{(0)}(q, \omega)$ (15) is large in the region of $(q, \omega)$ defined by the two conditions: 1$)$ the neutrino energy, $E_{1}$, is near the neutrino Fermi surface; 2) the electron energy, $E_{1}-\omega$, is near the electron Fermi surface. These conditions come from the occupation factors that enter the expression for the total rate.

In this region, we use (9), (10), and (11) to calculate the modification factors in the medium. We have estimated the contribution of the tensor term (11) relative to the two other terms and conclude that it is less than $10 \%$ of the total in the cores of protoneutron stars and supernovae. For the vector and axial-vector terms, we compute suppression factors, $S_{A}$ and $S_{V}$, defined as the ratio of the rates calculated with the nuclear interactions to those calculated without. This is done by substituting (9) and (10) into (5), multiplying (2) by the neutrino occupation function, and integrating over neutrino energies. In Table 1, we give results using a post-bounce supernova profile taken from Burrows, Hayes, \& Fryxell [8]. As seen in the table, the Gamow-Teller suppression factors are larger than the Fermi suppression factors. Furthermore, the degree of many-body suppression increases 
with density and decreases with temperature. Importantly, the magnitude of the effect above $10^{14} \mathrm{~g} \mathrm{~cm}^{-3}$ is large, ranging from a factor of 2 at $10^{14} \mathrm{~g} \mathrm{~cm}^{-3}$ to a factor of $\sim 5$ near $4 \times 10^{14} \mathrm{~g} \mathrm{~cm}^{-3}$. Correspondingly, the neutrino-matter absorption cross sections decrease with density. Since it has recently been shown [1] that the neutral-current scattering rates at high density are also reduced, we conclude that post-bounce supernova cores are significantly more transparent than previously believed. This enhanced transparency should translate at late times (> 500 - $2500 \mathrm{~ms}$ after bounce) into higher neutrino luminosities, that thereby may be more efficient at reenergizing or powering a stalled supernova shock [1].

Integrating equation (2) over $\cos \theta$, the distribution of the energy transfer, $\omega$, to and from the nucleons due to the process $\nu_{e}+n \rightarrow e^{-}+p$ in the lepton-rich era can be derived and is depicted in Figure 1 for a variety of densities, from $10^{13} \mathrm{~g} \mathrm{~cm}^{-3}$ to $10^{15} \mathrm{~g} \mathrm{~cm}^{-3}$. For these curves, the temperature is $5 \mathrm{MeV}$, the electron fraction, $\mathrm{Y}_{e}$, is 0.26 , the incident electron neutrinos are on their Fermi surfaces, and beta equilibrium is assumed. The highest curve on the right (that for $\rho=10^{13} \mathrm{~g} \mathrm{~cm}^{-3}$ ) ignores many-body effects, though it incorporates the full kinematics, and is included for comparison. As expected, the peak of the energy transfer is generally near $-\hat{\mu}$ (given in the figure caption), since the electron blocking factor in (2) puts the electrons on the electron Fermi surface and beta equilibrium requires that $\mu_{e}=\hat{\mu}+\mu_{\nu_{e}}$. (Note that $\hat{\mu}$ increases with density.) There is a modest spread in $\omega$ around the peak with approximately a gaussian distribution. The width of this distribution scales with the temperature. Figure 1 demonstrates what Table 1 also reflects that the total cross sections, the integrals under the unnormalized curves, are decreasing functions of density.

\section{LEPTON-POOR ERA}

As discussed in 9] and [10], as the trapped electron lepton number decays, we reach a configuration in which the neutrino absorption process discussed above dies almost completely for low temperatures. At the end of deleptonization, we have $p_{F}^{(\nu)}=0$ [0]. The neutrinos then have thermal energies and we find that the proton fraction has decreased

to the point that $2 p_{F}^{(p)}<<p_{F}^{(n)}$. In this case we cannot conserve momentum for the three degenerate species, $e^{-}, p, n$, when the momentum of the neutrino is small and when we stay near the Fermi surface for the three other species. At low temperatures, the function $\operatorname{Im} \Pi^{(0)}(q, \omega)$ of $(\sqrt{15})$ is now exponentially small in the region of $(q, \omega)$ defined by the leptonic occupancies. Thus, the ring graphs, as defined in the previous section, give a negligible rate for the charged-current processes at low lepton number and temperature.

The other mechanisms that have been used to estimate the rates depend on a spectator nucleon to transfer the necessary momentum, either through a potential or through an assumed correlation [9-11]. Translated to graphs, these mechanisms involve the estimation of proper graphs for the polarization parts, where a proper graph is defined as a graph that cannot be cut into two disjoint parts by severing a single potential line. However, there are "ring corrections" to such graphs, in which the initial or final current vertices attach to a ring chain that then attaches to the proper polarization graphs. These ring corrections then have a big suppressive effect on the primary mechanism for momentum transfer, as one can see from the following argument.

We consider a proper polarization graph in which a nucleon-nucleon interaction has intervened to allow momentum conservation near the Fermi surfaces. That is to say, by 
(10), we have a term for the vector contribution, $W_{V}$, to the rate formula (5) that has a substantial imaginary part in the kinematically-allowed region. We call this term, $W_{V}^{(1)}$. We now take the sum of this term and the lowest order term, $\Pi^{(0)}$, as the proper polarization part from which to construct the ring sum, obtaining,

$$
\operatorname{Im} W_{V}(q, \omega) \approx \frac{\operatorname{Im} W_{V}^{(1)}(q, \omega)}{\left|1-2 v_{1}\left[\Pi^{(0)}(q, \omega)+\operatorname{Re} W_{V}^{(1)}+i \operatorname{Im} W_{V}^{(1)}(q, \omega)\right]\right|^{2}} .
$$

In the estimates that follow, we omit the real part of $W_{V}^{(1)}$ from the denominator; it is easy to verify from (16) that, in contrast to the lowest-order imaginary part, the real part is not suppressed in the region of $(q, \omega)$ that is important in the reaction.

Similar considerations hold for the axial-vector part. In the lepton-poor era, we can drop the second term on the RHS of (5), since $\omega$ will be of the order of $T$, rather than of order 100-200 MeV, as it can be for electron neutrinos in the trapped neutrino era [12]. Then, we define a proper $W_{A}$ and a contribution $W_{A}^{(1)}$ as in the above and write

$$
\operatorname{Im} W_{A}(q, \omega) \approx \frac{\operatorname{Im} W_{A}^{(1)}(q, \omega)}{\left|1-2 v_{2}\left[\Pi^{(0)}(q, \omega)+i \operatorname{Im} W_{A}^{(1)}(q, \omega)\right]\right|^{2}} .
$$

For the factors $\operatorname{Im} W_{V, A}^{(1)}$ we take the minimal form consistent with avoiding a singularity in (2) at $\omega=-\hat{\mu}$ :

$$
\operatorname{Im} W_{V, A}^{(1)}=c_{V, A}^{(1)}(\omega+\hat{\mu}) .
$$

This form meets the requirement for detailed balance that Im $\Pi$ be odd under the replacements, $\omega \rightarrow-\omega, \hat{\mu} \rightarrow-\hat{\mu}$. We take the parameters $c^{(1)}$ to be sufficiently small for the integrated suppression factor to be independent of the $\left[c^{(1)}\right]^{2}$ that enter through the imaginary parts in the denominator function. In Table 2 we give, for the case of matter at nuclear density, the separate suppression factors for the vector and axial-vector rates generated by the uncorrected terms (19), under deleptonized conditions, $\mu_{\nu}=0$. We note that the reductions are substantial, and conclude that the ring corrections should be added to any model that is used for the neutrino opacities during this era.

\section{CONCLUSIONS}

We have developed a new formalism for incorporating the effects of many-body correlations on the charged-current rates of neutrino-matter interactions. This formalism reveals that these rates are considerably suppressed in the densest regions of protoneutron stars and supernova cores. Assuming that the nucleons are non-relativistic, our formalism incorporates the full kinematics of the interaction, Pauli blocking by final-state nucleons (protons), and correlation due to nucleon-nucleon interactions.

We have employed the ring approximation (RPA) and assumed the near-validity of Fermi Liquid Theory. It would desirable to include ladder diagrams and to perform the calculations in the context of a better numerical method for solution of the nuclear equation of state (EOS), since the solution of the EOS is intimately related to the derivation of the 
scattering/absorption rates. However, those who perform detailed nuclear EOS calculations and address many-body correlations in nuclear matter do not as yet provide the requisite spin and density structure functions, even for the static case.

These results for charged currents, when combined with the results from Burrows \& Sawyer [1] for neutral currents, strongly suggest that energy and lepton number will leak from supernova cores at a rate that is higher than heretofore estimated. This implies that the neutrino luminosities during the epoch after bounce for which the inner core is the major energy source $(>0.5-1.5 \mathrm{~s})$ will be enhanced, perhaps by as much as $50 \%$ [1]. The consequences of this increased transparency for the neutrino-driven supernova explosion mechanism [8] may be interesting, but have yet to be clarified.

\section{ACKNOWLEDGMENTS}

We thank S. Reddy, M. Prakash, G. Raffelt, and J. Lattimer for sharing their perspectives on this class of problems and acknowledge the support of the NSF under grant No. AST96-17494. We would also like to express our appreciation to the Santa Barbara Institute for Theoretical Physics, supported by the NSF under grant No. PHY94-07194. 


\section{TABLES}

TABLE I. The total suppression factors $\left(\mathcal{S}_{\mathcal{A}, \mathcal{V}}\right)$ for the process $\nu_{e}+n \rightarrow e^{-}+p$, for a profile in an early post-bounce model generated by Burrows, Hayes, \& Fryxell (1995). The suppression factors for the vector and the axial-vector terms are shown separately. These suppresion factors are derived by multiplying the rate by the neutrino occupation function and integrating over neutrino energies. A nucleon effective mass of $0.75 \times m_{n}$ is assumed.

\begin{tabular}{lccccc}
\hline \hline$\rho\left(\mathrm{g} \mathrm{cm}^{-3}\right)$ & $Y_{\nu}$ & $\mathrm{T}(\mathrm{MeV})$ & $Y_{e}$ & $\mathcal{S}_{\mathcal{A}}$ & $\mathcal{S}_{\mathcal{V}}$ \\
\hline $3.94 \times 10^{14}$ & 0.077 & 5 & 0.289 & 0.140 & 0.269 \\
$3.68 \times 10^{14}$ & 0.078 & 5 & 0.294 & 0.144 & 0.275 \\
$3.08 \times 10^{14}$ & 0.077 & 5 & 0.297 & 0.157 & 0.291 \\
$1.65 \times 10^{14}$ & 0.064 & 10 & 0.275 & 0.228 & 0.381 \\
$2.66 \times 10^{13}$ & 0.01 & 15 & 0.282 & 0.670 & 0.775 \\
$1.40 \times 10^{13}$ & 0.067 & 15 & 0.258 & 0.790 & 0.840 \\
\hline \hline
\end{tabular}

TABLE II. The total axial and vector suppression factors $\left(\mathcal{S}_{\mathcal{A}, \mathcal{V}}\right)$ for the transformation $\nu_{e} \rightarrow e^{-}$ during the lepton-poor era, for a density of $2.5 \times 10^{14} \mathrm{~g} \mathrm{~cm}^{-3}$ and a neutrino chemical potential of zero. These suppression factors are derived by multiplying the rate by the neutrino occupation function and integrating over neutrino energies. A nucleon effective mass of $0.75 \times m_{n}$ is assumed. See the text for details.

\begin{tabular}{cccc}
\hline \hline $\mathrm{T}(\mathrm{MeV})$ & $Y_{e}$ & $\mathcal{S}_{\mathcal{A}}$ & $\mathcal{S}_{\mathcal{V}}$ \\
\hline 3 & 0.012 & 0.34 & 0.53 \\
5 & 0.013 & 0.20 & 0.38 \\
7 & 0.016 & 0.19 & 0.38 \\
9 & 0.018 & 0.20 & 0.38 \\
\hline \hline
\end{tabular}




\section{REFERENCES}

[1] A. Burrows \& R.F. Sawyer, Phys. Rev. C, in press (1998).

[2] R. F. Sawyer, Phys. Rev. C40, 865 (1989).

[3] N. Iwamoto \& C. J. Pethick, Phys. Rev. D25, 313 (1982).

[4] G.E. Brown \& M. Rho, Nucl. Phys. A 372, 397 (1981).

[5] A.L. Fetter \& J.D. Walecka, Quantum Theory of Many Particle Systems (New York: McGraw-Hill), 1971.

[6] S. Reddy, M. Prakash, \& J.M. Lattimer, Phys. Rev. D, in press (1998).

[7] A. Burrows \& J. M. Lattimer, Astrophys. J. 307, 178 (1986).

[8] A. Burrows, J. Hayes, \& B.A. Fryxell, Astrophys. J. 450, 830 (1995).

[9] R. F. Sawyer \& A. Soni, Astrophys. J. 230, 859 (1979)

[10] P. Haensel \& A. J. Jerzak, Astron. Astrophys. 179, 127 (1987).

[11] B.L. Friman \& O.V. Maxwell, Astrophys. J. 232, 741 (1979).

[12] Note that, as in [10], a tensor force can nonetheless be the dominant ingredient in creating a proper polarization part that has a significant imaginary part in the region of interest, for either the vector or axial--vector current parts. 


\section{FIGURES}

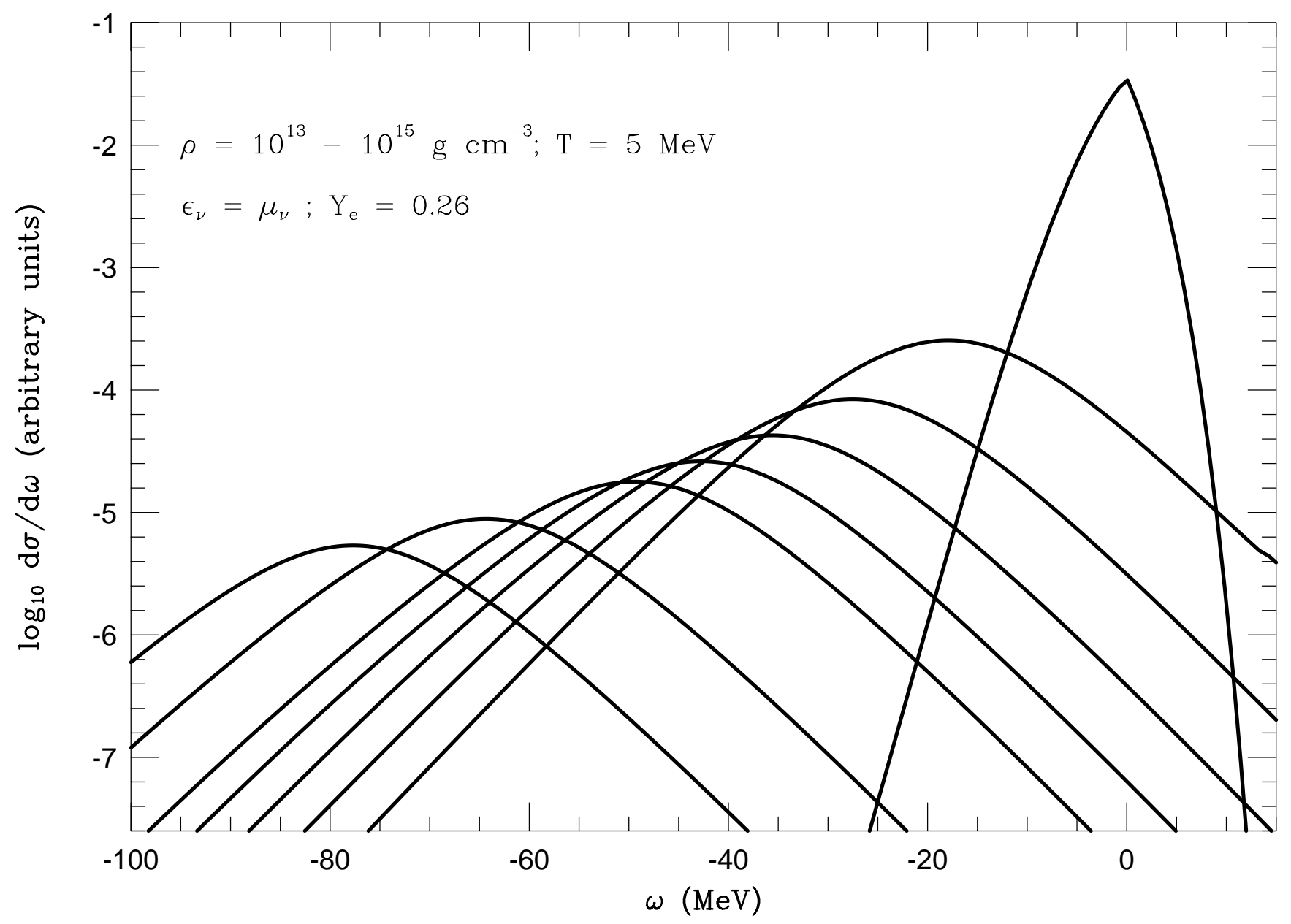

FIG. 1. The singly-differential cross section in arbitrary units versus the energy transfer, $\omega$, to the nucleons due to the process $\nu_{e}+n \rightarrow e^{-}+p$ in the lepton-rich era. $\omega$ is in $\mathrm{MeV}$. We have integrated Eq. (2) over $\cos \theta$. The curves are for mass densities of $10^{13}, 10^{14}, 2 \times 10^{14}, 3 \times 10^{14}$, $4 \times 10^{14}, 5 \times 10^{14}, 7.5 \times 10^{14}$, and $10^{15} \mathrm{~g} \mathrm{~cm}^{-3}$. The temperature is $5 \mathrm{MeV}$, the electron fraction is 0.26 , the incident electron neutrinos are on their Fermi surfaces, and beta equilibrium has been assumed. The $10^{13} \mathrm{~g} \mathrm{~cm}^{-3}$ curve does not include many-body effects, but does incorporate the full kinematics. For the other curves, $v_{2}=4.5 \times 10^{-5}(\mathrm{MeV})^{-2}, v_{1}=1.76 \times 10^{-5}(\mathrm{MeV})^{-2}$, and an effective mass of $0.75 \times \mathrm{m}_{n}$ was assumed. $\hat{\mu}$ is equal to $5.3,17.4,26.8,34.8,42.1,48.7,63.7$, $77.1 \mathrm{MeV}$ for the densities depicted. 